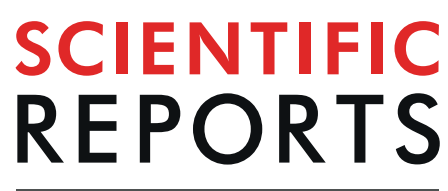

natureresearch

Received: 14 May 2019

Accepted: 1 August 2019

Published online: 12 August 2019

\section{Genome-wide Identification, Classification, Expression and Duplication Analysis of GRAS Family Genes in Juglans regia L.}

\author{
Shaowen Quan ${ }^{1,2}$, Jianxin Niư,2, Li Zhou ${ }^{1,2}$, Hang Xu ${ }^{1,2}$, Li Ma ${ }^{1,2}$ \& Yang Qin ${ }^{1,2}$
}

Fifty-two GRAS genes are identified in walnut genome. Based on the evolutionary relationship and motif analysis, the walnut GRAS gene family was divided into eight subfamilies, and the sequence features analysis of JrGRAS proteins showed that the JrGRAS protein sequences were both conserved and altered during the evolutionary process. Gene duplication analysis indicated that seven GRAS genes in walnut have orthologous genes in other species, and five of them occurred duplicated events in walnut genome. Expression pattern analysis of the GRAS family genes in walnut showed that two JrGRAS genes ( $J r C I G R a-b$ and JrSCL28a) were differentially expressed between flower bud and leaf bud $(\mathrm{p}<0.01$ ), and two JrGRAS genes (JrCIGRa-b and JrSCL13b- $d$ ) were differentially expressed between the different development stages of flower buds transition $(\mathrm{p}<0.01)$, besides, three hub genes (JrGAla, $J r S C L 3 f$ and JrSHRc) were identified by co-expression analysis, which suggested these GRAS genes may play an important role in regulating the development of apical meristem in walnut. This study laid a foundation for further understanding of the function of GRAS family genes in walnut.

GRAS genes, derived from the first three members to be identified as a plant-specific gene family, the GIBBERELLIN-INSENSITIVE (GAI), Repressor of ga1-3 (RGA) and SCARECROW $(S C R)^{1}$. Among them, GAI proteins and RGA proteins are members of the DELLA proteins, which play important roles in repressing gibberellin responses ${ }^{2}$ and jasmonate (JA) and light signaling regulation ${ }^{3}$, and SCR proteins act as a key regulator of Arabidopsis roots ${ }^{4-6}$.

GRAS proteins share conserved domains in their C-terminus, comprised LHR I, VHIID, LHR II, PFYRE and SAW ${ }^{1,7-9}$, however, the $\mathrm{N}$-terminus of GRAS proteins show a great divergence, which may result to the functional specificity of each protein ${ }^{10}$. Although metazoan STATs share similar domain organization with plant GRAS, it is lack of enough support for the hypothesis that GRAS proteins are plant STATS ${ }^{11}$. Recent structural studies have illustrated that the conserved GRAS domain comprises an $\alpha$-helical cap and $\alpha / \beta$ core subdomains, which mediates protein-protein interactions ${ }^{4}$.

Up to now, more than a dozen of GRAS gene family have been identified, including Arabidopsis thaliana $a^{1,7,12}$, Rice $^{7,13}$, Populus ${ }^{14}$, pine ${ }^{15}$, Chinese cabbage ${ }^{16}$, tobacco ${ }^{17}$, tomato $^{18,19}$, Prunus mume ${ }^{20}$, Jatropha curcas L. $^{21}$, Lotus japonicus ${ }^{22}$, grapevine ${ }^{23,24}$, Nelumbo nucifera ${ }^{25}$, Ricinus communis ${ }^{26}$, Betula kirghisorum ${ }^{27}$, Isatis indigotica ${ }^{28}$, apple ${ }^{29}$, Zea mays L L. $^{30}$, Medicago truncatula ${ }^{31}$, Camellia sinensis ${ }^{32}$ and Gossypium hirsutum ${ }^{33}$. The plant-specific GRAS family of proteins function as transcriptional regulators and play critical roles in development and signa-

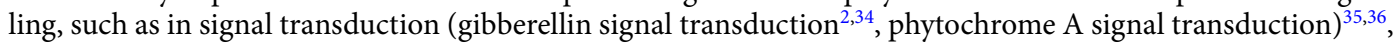
stress responses ${ }^{23,37-40}$, meristem formation and maintenance ${ }^{8,41-44}$ and promoting flowering ${ }^{9}$.

Walnut is cultivated worldwide for its nutritious fruits and commercially valuable timber, however, it needs many years before flowering and to become productive ${ }^{45-47}$. Previous research has shown that some of the GRAS members play important roles in meristem development ${ }^{8,41-44}$. To better understanding the molecular mechanism of walnut flower bud transition, it is necessary to investigate the GRAS family in walnut. With the availability of 
walnut genome sequences ${ }^{48}$ and transcriptome data of the walnut female flower buds and leaf buds, it is possible for us to identify all the GRAS family genes in walnut.

In this study, GRAS family genes in walnut have been identified in genome-wide. The phylogenetic relationship, sequence alignment, conserved motif composition and gene duplication of the JrGRAS genes were systematically analyzed, and their expression patterns in different tissues (flower bud and leaf bud) and different development stages (before, during, after the flower transition period) were explored using transcriptome data and validated by qRT-PCR experiments. Finally, protein-protein interactions analysis was conducted to investigate how they participate in diverse functions by interacting with other proteins. This research lay a foundation for further function investigations of GRAS genes in walnut.

\section{Results}

Identification of GRAS family members in walnut. A total of seventy protein sequences (include protein isoforms) encoded by fifty-two genes, which including the GRAS domain were identified as the walnut GRAS proteins for further analysis. Fifty-two GRAS genes locate in 44 scaffolds, and their start position and end position are shown in Table 1. The candidate GRAS members were then uploaded to the CD-search website (https://www. ncbi.nlm.nih.gov/Structure/cdd/wrpsb.cgi) and their domain information were listed in Table 1, too. Besides, the gene structures of JrGRAS was presented in Fig. S1, and subcellular location information of the JrGRAS proteins was presented in Table S1.

Phylogenetic analysis of GRAS members. To study the phylogenetic relationships between GRAS family members in walnut, domain sequences of 70 walnut GRAS proteins, 33 Arabidopsis GRAS proteins and 43 grape GRAS proteins were used to construct an unrooted NJ phylogenetic tree in MEGA 6 with 1000 bootstrap replicates (Fig. 1). Based on the phylogenetic analysis and previous research ${ }^{1}$, all GRAS members were clustered into 8 subfamilies: PAT1, SCL3, DELLA, LAS, SCR, HAM, SHR, LISCL. The distribution of JrGRAS proteins among different subfamilies was as following: PAT1(20), LISCL(14), DELLA (10), SCR(8), SCL3(6), HAM(4), LAS(4), and SHR(4).

Definition the sequence features of JrGRAS proteins. The GRAS proteins in walnut share a highly conserved C-terminal, which is constituted by five distinct conserved motifs in the following order: LHR I (leucine heptad repeat I), VHIID, LHR II (leucine heptad repeat II), PFYRE and SAW, while the N-terminal region of the sequences seems to be variable (Fig. 2).

The presence of leucine heptad repeats in the GRAS proteins suggests that these proteins may function as multimers and a potentially complicated higher order of interaction ${ }^{1}$. The VHIID sequence consists of valine, histidine, isoleucine and aspartic acid, which is not absolutely conserved although it can be readily recognizable (position: 214-218, Fig. 2). Besides, we noticed the VHIID motif, the P residues (position: 191, Fig. 2) are absolutely conserved in the VHIID motif. The PFYRE motif consists of the P(position: 342$)-F$ (position: 363)-Y(position: 374)-R(position: 366)-E(position: 369) (Fig. 2) residues, the $P$ residues are absolutely conserved in PFYRE motif as well as in motif VHIID. The SAW motif is characterized by the residues S-A-W (position: 481483, Fig. 2), the W(position: 472,483) residues are absolutely conserved in the other JrGRAS protein sequences, except the JrSLR1 which lack the SAW motif. And the absolute conservation of the residues in the VHIID and SAW motifs indicates that these residues could be necessary for the functions of the GRAS proteins.

Conserved motifs analyses. All JrGRAS proteins were subjected to MEME website (http://meme-suite. $\mathrm{org} /$ tools $/ \mathrm{meme})^{49}$ to identify conserved motifs (Fig. 3). Among the twenty Motifs, Motifs 10 and 4 consisted the LHR I domain, Motifs 1 and 8 consisted the VHIID domain, Motifs 6,9 and 17 or 6 and 20 consisted the LHR II domain, Motifs 7,3 and 19 consisted the PFYRE domain, and Motifs 2, 16 and 5 or 14 and 5 consisted the SAM domain (Fig. 3). Interesting, almost all JrGRAS protein include the complete GRAS motif model, which consists of LHR I, VHIID, LHR II, PFYRE, and SAM domain, and the five domains distribute in the same order, except $J r S L R 1, J r S C L a$ and JrSCLf.

Synteny analysis and gene duplication of JrGRAS genes. Synteny analysis between different species. To deduce the evolutionary relationship of GRAS genes between different species, syntenic analysis was performed for three plants (A. thaliana, Vitis vinifera and Juglans regia) (Fig. 4A). The result showed that there are many synteny blocks between Arabidopsis, grape and walnut. Among these blocks, seven walnut GRAS genes ( $[$ rGAIb/JrSCL22b/IrSCL28b-c/IrSCL15/JrSCL14b/IrSCL9/JrSCL28a) showed pairwise synteny with genes in Aradiposis genome, and twenty-one walnut GRAS genes (JrSCL27/JrSCL22a/JrSCL3a/JrGAIb/JrSCL21a-d/JrSC

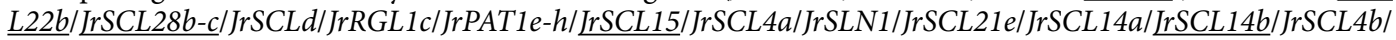
JrCIGRa-b/【rSCL9/JrNSP2a/IrSCL28a) showed pairwise synteny with genes in grape genome. What is more, the seven walnut GRAS genes (IrGAIb/IrSCL22b/IrSCL28b-c/IrSCL15/IrSCL14b/IrSCL9/IrSCL28a) were identified to have orthologous genes within Aradiposis genome and within grape genome, simultaneously. These data indicated that the GRAS genes might have evolved from the common ancestor in different plants (The gene name with an underline means this gene was identified as the orthologous gene between different species).

Gene duplication in walnut genome. Gene duplication events were surveyed to explore the evolutionary patterns of the GRAS gene family in walnut genome (Fig. 4B). Physical locations of $52 \mathrm{JrGRAS}$ genes in walnut were investigated by analysis of genomic distribution on scaffolds. Fifty-two JrGRAS genes were distributed unevenly across the 44 scaffolds in the walnut genome (Fig. 4B). Analysis of walnut GRAS family genes revealed seven paralogous gene pairs (JrGAIa\&LrGAIb/JrRGL1c\& JrSCl21e/IrSCL14b\&JrSCL34a/IrSCL22b\&JrSCL27/IrSCL28a\&IrSCL28b-c/ JrSCL3c\&JrSCL3d-e/JrSCL4a\&JrSCL4b) existed in walnut GRAS family genes. Among the 14 GRAS paralogous 


\begin{tabular}{|c|c|c|c|c|c|c|c|c|}
\hline Gene name & Gene symbol & Scaff & Scaff rename & Genome location & Strand & Related protein & $\begin{array}{l}\text { Protein short } \\
\text { name }\end{array}$ & $\begin{array}{l}\text { GRAS domain } \\
\text { position }\end{array}$ \\
\hline JrRGL1a & LOC108981380 & NW_017389446.1 & Scaff13 & NW_017389446.1: 52316-54604 & - & XP_018808049.1 & JrRGL1a & $155-526$ \\
\hline JrSCL28a & LOC108984412 & NW_017442835.1 & Scaff27 & NW_017442835.1: 99273-101699 & + & XP_018811904.1 & JrSCL28a & $303-669$ \\
\hline \multirow{2}{*}{$J r S C L 1 a-b$} & \multirow{2}{*}{ LOC108984751 } & \multirow{2}{*}{ NW_017443020.1 } & \multirow{2}{*}{ Scaff30 } & \multirow{2}{*}{ NW_017443020.1: 188763-190597 } & \multirow{2}{*}{-} & XP_018812342.1 & JrSCL1a & $202-571$ \\
\hline & & & & & & XP_018812343.1 & JrSCL1b & $202-571$ \\
\hline \multirow{3}{*}{ JrSCL21a-d } & \multirow{3}{*}{ LOC108985037 } & \multirow{3}{*}{ NW_017439731.1 } & \multirow{3}{*}{ Scaff20 } & \multirow{3}{*}{ NW_017439731.1: 8737-10242 } & \multirow{3}{*}{-} & XP_018812737.1 & $J r S C L 21 b$ & $176-546$ \\
\hline & & & & & & XP_018812742.1 & JrSCL21c & $176-546$ \\
\hline & & & & & & XP_018812749.1 & JrSCL21d & $176-546$ \\
\hline JrSCL23a & LOC108985505 & NW_017443560.1 & Scaff36 & NW_017443560.1: 1461510-1465205 & + & XP_018813375.1 & JrSCL23a & $73-427$ \\
\hline JrSCL14a & LOC108986374 & NW_017389857.1 & Scaff15 & NW_017389857.1: 63443-68155 & + & XP_018814536.1 & JrSCL14a & $369-740$ \\
\hline JrNSP2a & LOC108988310 & NW_017442823.1 & Scaff26 & NW_017442823.1: 505495-509211 & + & XP_018817086.1 & $J r \mathrm{NSP} 2 \mathrm{a}$ & $117-501$ \\
\hline JrSHRa & LOC108988543 & NW_0174435543.1 & Scaff34 & NW_017443543.1: 934500-937558 & + & XP_018817374.1 & JrSHRa & $105-484$ \\
\hline$J r S C L 3 b$ & LOC108988679 & NW_017389863.1 & Scaff16 & NW_017389863.1:241932-244037 & + & XP_018817550.1 & $J r S C L 3 b$ & $44-457$ \\
\hline JrRGL1b & LOC108989561 & NW_017389020.1 & Scaff10 & NW_017389020.1: 713954-717041 & - & XP_018818751.1 & JrRGL1b & $138-508$ \\
\hline JrSCL27 & LOC108990734 & NW_017389863.1 & Scaff16 & NW_017389863.1:270998-273865 & - & XP_018820345.1 & JrSCL27 & $379-740$ \\
\hline JrSCL18 & LOC108992395 & NW_017389020.1 & Scaff10 & NW_017389020.1: 724451-727425 & - & XP_018822504.1 & JrSCL18 & $47-445$ \\
\hline JrSHRb & LOC108992438 & NW_017389863.1 & Scaff16 & NW_017389863.1:265516-268446 & - & XP_018822539.1 & JrSHRb & $60-433$ \\
\hline JrSCL14b & LOC108992934 & NW_017389020.1 & Scaff10 & NW_017389020.1: 761280-765445 & + & XP_018823200.1 & JrSCL14b & $325-701$ \\
\hline JrSCL13a & LOC108993395 & NW_017443546.1 & Scaff35 & NW_017443546.1: 1236377-1240941 & + & XP_018823840.1 & JrSCL13a & $176-546$ \\
\hline JrPAT1a-d & LOC108994062 & NW_017443598.1 & Scaff41 & & & XP_018824686.1 & JrPAT1a & $168-538$ \\
\hline MOLLE-c & LUC100995090 & NW $-01 / 20000 \%$ & ocallt & NWV-01750000/.1. 1535250-1530541 & - & XP_018827111.1 & $J r S C L c$ & $438-790$ \\
\hline JrSCLd & LOC108995938 & NW_017388969.1 & Scaff8 & NW_017388969.1: 382640-385001 & + & XP_018827159.1 & JrSCLd & $152-507$ \\
\hline JrSCL23b & LOC108996381 & NW_017388856.1 & Scaff1 & NW_017388856.1: 1066492-1069818 & - & XP_018827796.1 & $J r S C L 23 b$ & $73-427$ \\
\hline & & & & & & XP_018828372.1 & $J r S C L 13 b$ & $174-545$ \\
\hline$J r S C L 13 b-d$ & LOC108996812 & NW_017388861.1 & Scaff3 & NW_017388861.1: 936197-939325 & - & XP_018828373.1 & JrSCL13c & $174-545$ \\
\hline & & & & & & XP_018828374.1 & $J r S C L 13 \mathrm{~d}$ & $174-545$ \\
\hline $\operatorname{IrSCL} 28 h-c$ & $10 C 108997020$ & NW 0174427201 & Scaff25 & NW $0174427201 \cdot 50539-52384$ & - & XP_018828642.1 & JrSCL28b & $304-670$ \\
\hline $\operatorname{frscL} 28 b-c$ & 200108997020 & NW_01/442/20.1 & scallzs & $N W=01 / 442 / 20.1: 50539-52384$ & - & XP_018828643.1 & JrSCL28c & $304-643$ \\
\hline JrSCL4b & LOC108997571 & NW_017443591.1 & Scaff40 & NW_017443591.1: 871325-873488 & + & XP_018829455.1 & $J r S C L 4 \mathrm{~b}$ & $252-623$ \\
\hline 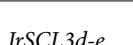 & IOC108999242 & NW 0173888931 & $S_{\text {caff5 }}$ & NW $0173888931 \cdot 2740337-2743452$ & + & XP_018831643.1 & JrSCL3d & $46-468$ \\
\hline Jrsclsa-e & LUC108999242 & NW_01/388893.1 & Scails & NW_-01/388893.1:2/4033/-2/45432 & + & XP_018831644.1 & JrSCL3e & $46-468$ \\
\hline JrRGL1c & LOC109001324 & NW_017442404.1 & Scaff23 & NW_017442404.1:246803-250094 & - & XP_018834108.1 & JrRGL1c & $310-676$ \\
\hline JrSCL21e & LOC109001839 & NW_017443600.1 & Scaff 42 & NW_017443600.1: 47744-51063 & - & XP_018834825.1 & $J r S C L 21 \mathrm{e}$ & $315-681$ \\
\hline JrSHRc & LOC109002462 & NW_017441391.1 & Scaff22 & NW_017441391.1: 33841-36019 & - & XP_018835769.1 & JrSHRc & $110-490$ \\
\hline $\operatorname{IrSCL} 33 a-b$ & LOC109002666 & NW 0174430091 & Scaff29 & NW $0174430091 \cdot 917876-919261$ & + & XP_018836065.1 & $J r S C L 33 a$ & $367-737$ \\
\hline $\operatorname{mrocL33a-b}$ & 201109002000 & NW_-01/445009.1 & scallzy & NW_01/443009.1:91/8/0-919201 & + & XP_018836066.1 & $J r S C L 33 b$ & $367-737$ \\
\hline JrSCL34a & LOC109002667 & NW_017388893.1 & Scaff5 & NW_017388893.1: 730620-732711 & + & XP_018836067.1 & JrSCL34a & $383-753$ \\
\hline JrSCL9 & LOC109002669 & NW_017443569.1 & Scaff37 & NW_017443569.1: 521973-524512 & + & XP_018836069.1 & JrSCL9 & $386-757$ \\
\hline JrSCLe & LOC109004170 & NW_017443629.1 & Scaff44 & NW_017443629.1: 436277-438894 & + & XP_018838179.1 & JrSCLe & $449-801$ \\
\hline JrSLN1 & LOC109006296 & NW_017437159.1 & Scaff19 & NW_017437159.1: 15109-18210 & + & XP_018841073.1 & JrSLN1 & $151-518$ \\
\hline JrGAIa & LOC109007807 & NW_017443578.1 & Scaff38 & NW_017443578.1: 1317343-1319989 & + & XP_018843202.1 & JrGAIa & $226-585$ \\
\hline
\end{tabular}




\begin{tabular}{|c|c|c|c|c|c|c|c|c|}
\hline Gene name & Gene symbol & Scaff & Scaff rename & Genome location & Strand & Related protein & $\begin{array}{l}\text { Protein short } \\
\text { name }\end{array}$ & $\begin{array}{l}\text { GRAS domain } \\
\text { position }\end{array}$ \\
\hline \multirow{2}{*}{ JrPAT1e-h } & \multirow{2}{*}{ LOC109012627 } & \multirow{2}{*}{ NW_017443590.1 } & \multirow{2}{*}{ Scaff39 } & \multirow{2}{*}{ NW_017443590.1: 1210837-1213906 } & \multirow{2}{*}{+} & XP_018849899.1 & JrPATlf & $176-546$ \\
\hline & & & & & & XP_018849901.1 & JrPATlh & $176-546$ \\
\hline$J r S C L 34 b$ & LOC109013013 & NW_017443590.1 & Scaff39 & NW_017443590.1: 1237365-1238906 & - & XP_018850468.1 & $J r S C L 34 \mathrm{~b}$ & $383-758$ \\
\hline $\operatorname{JrSCL} 33 \mathrm{c}-\mathrm{d}$ & LOC109013014 & NW_017389181.1 & Scaff11 & NW_017389181.1: 2256-5719 & - & XP_018850470.1 & JrSCL33c & $385-759$ \\
\hline JrSCL14c & LOC109013019 & NW_017417453.1 & Scaff18 & NW_017417453.1: 8-1329 & - & XP_018850477.1 & JrSCL14c & $328-699$ \\
\hline \multirow{2}{*}{$J r C I G R a-b$} & \multirow{2}{*}{ LOC109014308 } & \multirow{2}{*}{ NW_017443037.1 } & \multirow{2}{*}{ Scaff31 } & \multirow{2}{*}{ NW_017443037.1: 144211-146190 } & \multirow{2}{*}{-} & XP_018852274.1 & JrCIGRa & $207-576$ \\
\hline & & & & & & XP_018852283.1 & $J r \mathrm{CIGRb}$ & $207-576$ \\
\hline$J r S C L 22 b$ & LOC109015811 & NW_017443532.1 & Scaff33 & NW_017443532.1: 1336635-1338234 & + & XP_018853819.1 & $J r S C L 22 b$ & $386-747$ \\
\hline JrSLR1 & LOC109015902 & NW_017389006.1 & Scaff9 & NW_017389006.1: 187337-190408 & + & XP_018853896.1 & JrSLR1 & $151-449$ \\
\hline
\end{tabular}

Table 1. GRAS gene family identified in Juglans regia.

genes, 5 of them were orthologous genes identified between species, which indicated they were involved in the duplication event in walnut genome. (The gene name with an underline means this gene was identified as the orthologous gene between different species and the ' $\&$ ' means connector between duplicated gene pairs).

Expression profiles of GRAS members. We used the FPKM values of 52 JrGRAS genes to investigate the expression profiles of the JrGRAS family genes. Ten of the JrGRAS genes were excluded to draw the heatmap for their FPKM value were zero in both flower bud and leaf bud.

First, expression levels of JrGRAS genes in female flower bud and in leaf bud were compared (Fig. 5A). Three $J r G R A S$ genes ( $J r S C L 22 a / J r G A I b / J r G A I c$ ) were highly expressed in both flower bud and leaf bud, and four JrGRAS genes (JrSCL18/JrSCL32/JrRGL1d/JrPAT1a-d) were lowly expressed in both flower bud and leaf bud. Besides, two JrGRAS genes ( $J r C I G R a-b / J r S C L 28 a)$ were differentially expressed between flower bud and leaf bud $(\mathrm{p}<0.01)$.

Next, expression levels of the JrGRAS genes in female flower buds before, during, and after flower transition (F_1/F_2/F_3) were compared (Fig. 5B). Four JrGRAS genes (JrSCL18/JrSCL32/JrRGL1d/JrPAT1a-d) were lowly expressed in F_1, F_2 and F_3, and four JrGRAS genes (JrSCL15/JrSCL22a/JrGAIb/JrGAIc) were highly expressed in both flower bud and leaf bud. Besides, two JrGRAS genes (JrCIGRa- $b$ and JrSCL13b-d) were differentially expressed between F_1, F_2 and F_3 $(\mathrm{p}<0.01)$.

GO enrichment. The GO enrichment analysis based on the $70 \mathrm{JrGRAS}$ proteins annotated in the GO database. In the biological process category, significantly enriched terms were associated with biological regulation, cellular process, metabolic process, and response to stimulus. In the cellular component category, cell, cell part, and organelle were significantly enriched. In the molecular function category, GO terms related to binding and nucleic acid binding transcription factor activity were highly represented. Besides, GO: 003674 (molecular function) was the most GO term enriched by the JrGRAS members (Fig. S2).

Co-expression networks analysis of the JrGRAS family genes. Weighted gene co-expression network analysis (WGCNA) is a biology method for interaction analysis and correlation networks resolving ${ }^{50}$. To search for the genes involved in flowering time regulation in walnut, JrGRAS family genes were used to construct a co-expression network with the method of WGCNA, the result was presented in Fig. 6 . In the co-expression network, many of the key genes that participate in walnut flower bud transition were identified, such as JrGAIa, JrSCL3f, JrSHRc, JrSCL34a, JrSLR1, JrRGL1d, JrSLN1, JrSCL18 and the hub genes with the highest edge numbers were JrGAIa, JrSCL3f and JrSHRc.

Validation expression patterns of JrGRASs by qRT-PCR. The top five JrGRAS genes (JrSCL3f/JrSHRc/JrGAIa/JrSLN1/JrRGL1d) in the co-expression network and three DEGs (JrCIGRa-b, JrSCL13b-d and $J r S C L 28 a$ ) were used to conduct a qRT-PCR experiment (Fig. 7). The results were similar to those of our RNA-seq analysis and the DEGs were evidently differentially expressed among different tissues and development stages $(\mathrm{P}<0.01)$. In leaf bud, JrCIGRa- $b$ and $J r S C L 28 a$ were all significantly up-regulated than that in flower bud $(\mathrm{P}<0.01)$. As for flower bud transition periods, JrCIGRa-b and JrSCL13b-d were up-regulated in F_3 than that in $\mathrm{F} \_1$ and $\mathrm{F} \_2(\mathrm{P}<0.01)$. Among the DEGs, JrCIGR $a$ - $b$ differentially expressed in different tissues and different development period of flower buds, suggesting that this gene should work as the candidate gene for flower bud transition in walnut.

Interaction network of JrGRAS proteins. Because the interaction of walnut GRAS proteins is little known, we constructed the interaction network of the JrGRAS proteins based on interaction relationship of the homologous GRAS proteins in Arabidopsis. The walnut GRAS proteins corresponded with the Arabidopsis 


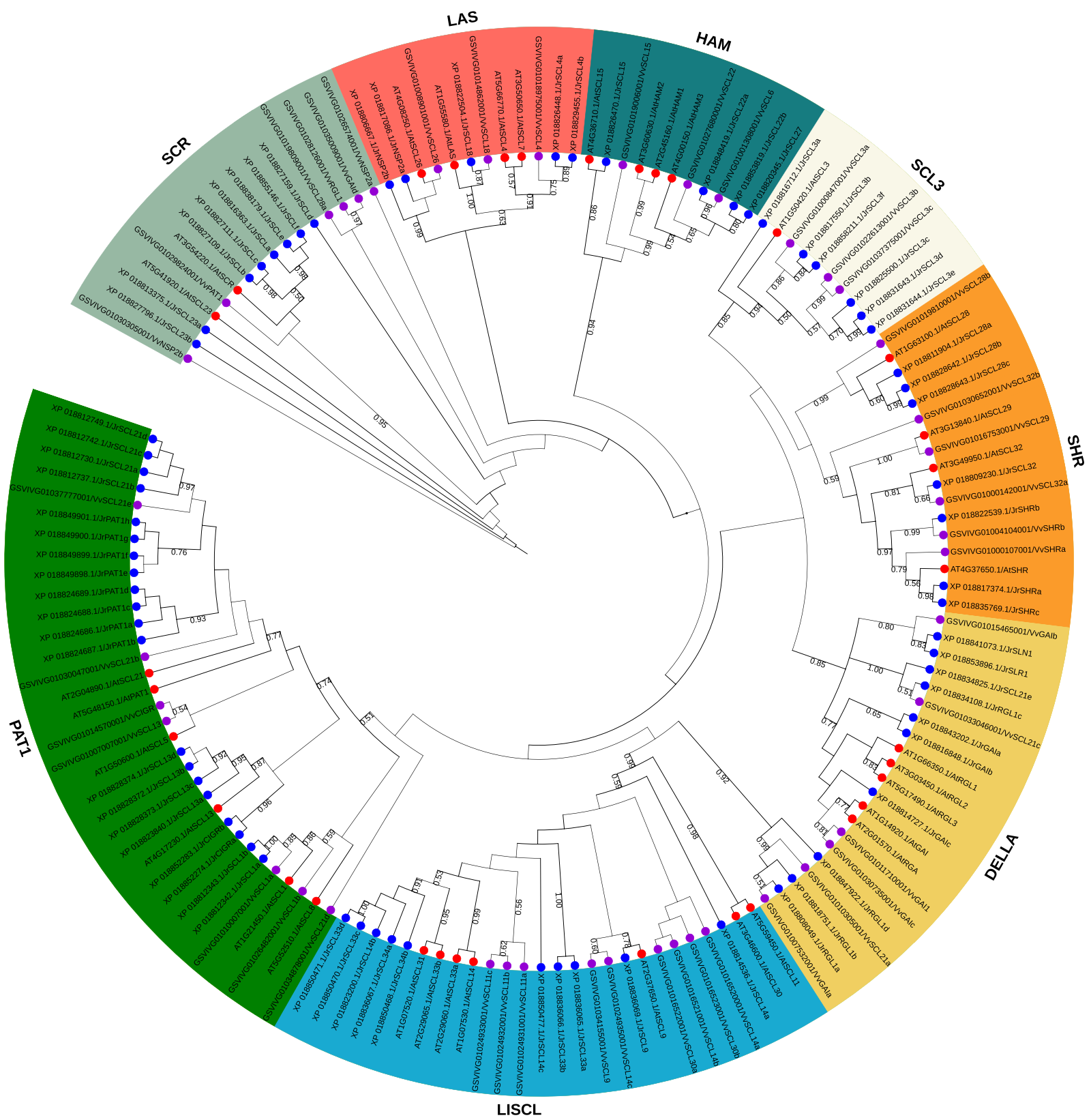

Figure 1. Phylogenetic tree of the domain sequence of GRAS proteins from Arabidopsis, walnut and grape using the Maximum Likelihood method. Genes in Arabidopsis, walnut and grape are labeled in red, blue and purple dots, respectively.

GRAS proteins are listed below them (Fig. 8). The result showed that several JrGRASs (such as JrGAIb/JrGAIc) were predicted to be core nodes in the network, which suggested that they might participate in diverse functions by interacting with other proteins.

\section{Discussion}

In general, analysis of whole genome location and evolution rely on the available information of species genome assembled in Chromosomes-level. However, the walnut genome was assembled only in scaffold-level, and there is no access to the information of walnut Chromosomes until now. In this article, the 44 scaffolds which including the 52 JrGRAS genes were used to represent the walnut genome in the synteny and gene duplication analysis, and this may provide a new insight to the analysis of whole genome evolution for the species whose genome assembled in scaffold-level.

Evolution of divergence and conservation. Divergence and conservation always come together with the process of species evolution. Phylogenetic analysis divided the JrGRAS family into eight subgroups based on the evolutionary relationship, and each subgroup always function differently (Fig. 1). However, sequence alignment 

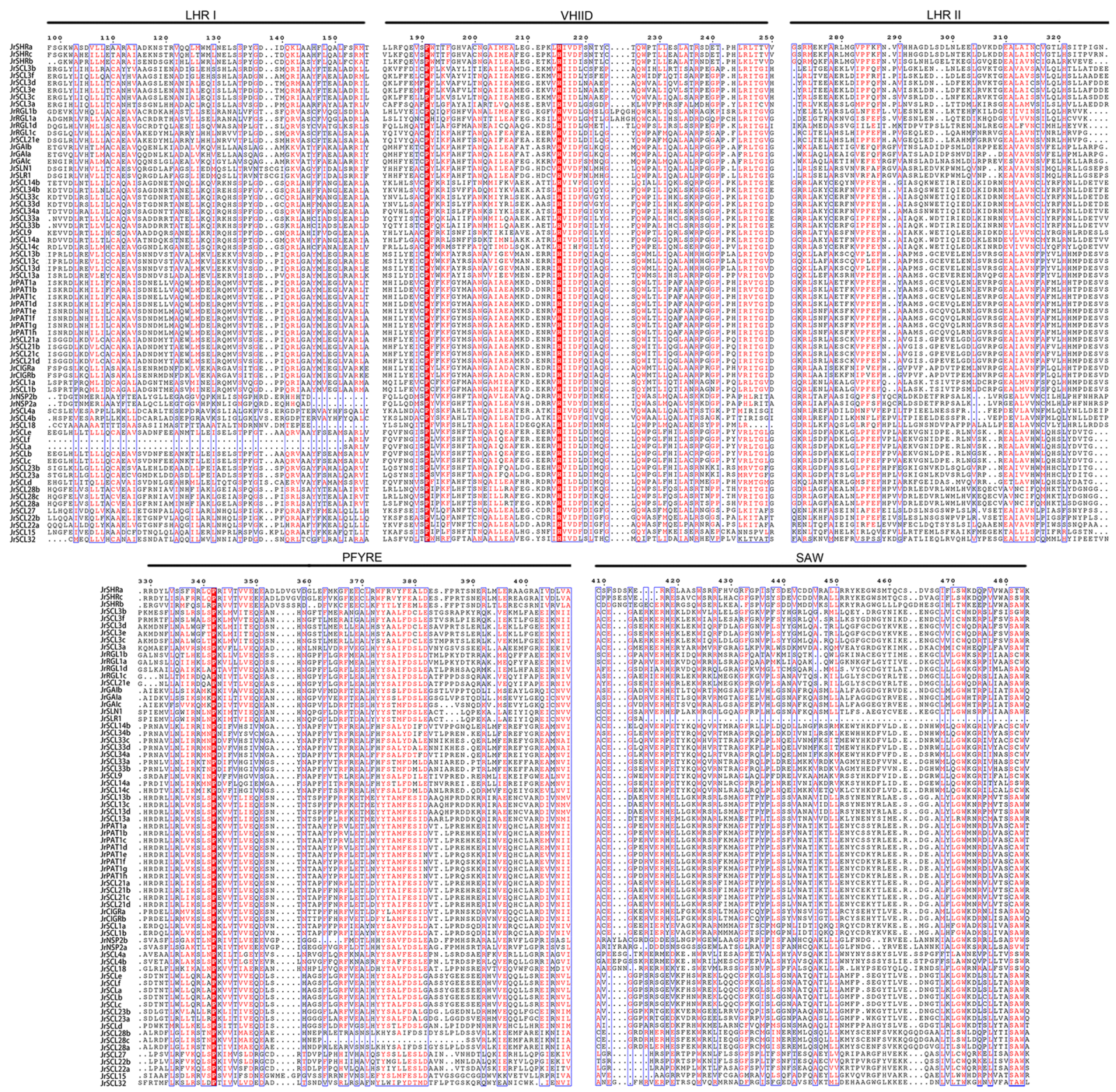

Figure 2. Alignment of the walnut GRAS protein sequences. The highly conserved regions of the JrGRAS proteins were divided into five recognizable motifs.

indicated that it was high conserved for the distribution of five motifs (LHR I, VHIID, LHR II, PFYRE, SAW motif) in $J r G R A S$ family members, and the order of these motifs within each protein is the same (Figs 2 and 3 ). Besides, in VHIID and SAW motifs, the absolute conserved residues suggested that these residues could be necessary for the activity of the GRAS proteins (Fig. 2).

The duplication of GRAS genes between species and in walnut genome. Gene duplication between species indicated that Arabidopsis, walnut, and grape share the same seven ancestral GRAS genes. The number of orthologous genes of GRAS family genes in the three species showed a ratio of 7:21:21 (Arabidopsis: walnut: grape), which suggest a triplication event could occur in the GRAS family gene of walnut and grape. These caused us to further investigate the expansion of GRAS family gene in the walnut genome.

However, duplication analysis in walnut genome indicated that the triplicated speculation was invalid. Besides, duplicate genes face fates as follow: non-functionalization, neo-functionalization (evolving novel functions), or sub-functionalization (partition of gene functions $)^{51}$. The seven orthologous GRAS genes (IrGAIb/JrS

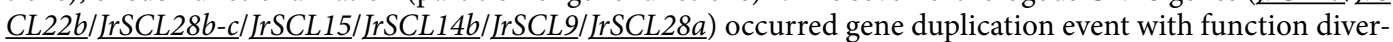
gent in walnut genome, five of them duplicated with their pair genes (JrGAIb\&JrGAIa/JrSCL22b\&JrSCL27/JrSCL

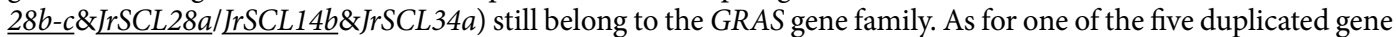

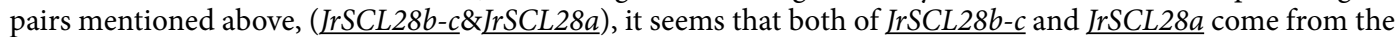
orthologous GRAS genes and this suggests that the duplication of them could be earlier than that in the other four 


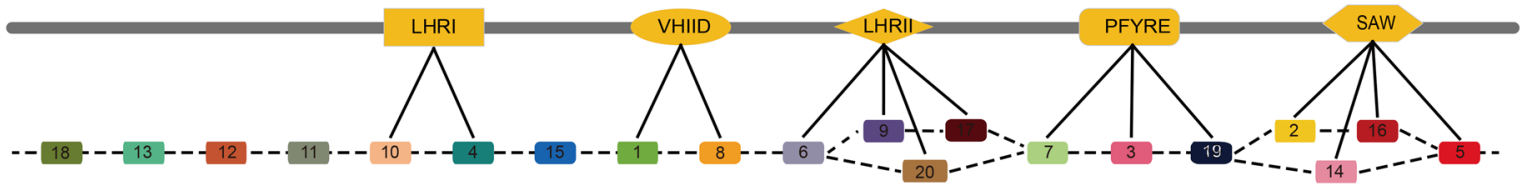

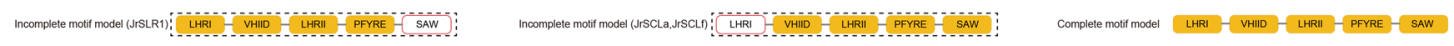

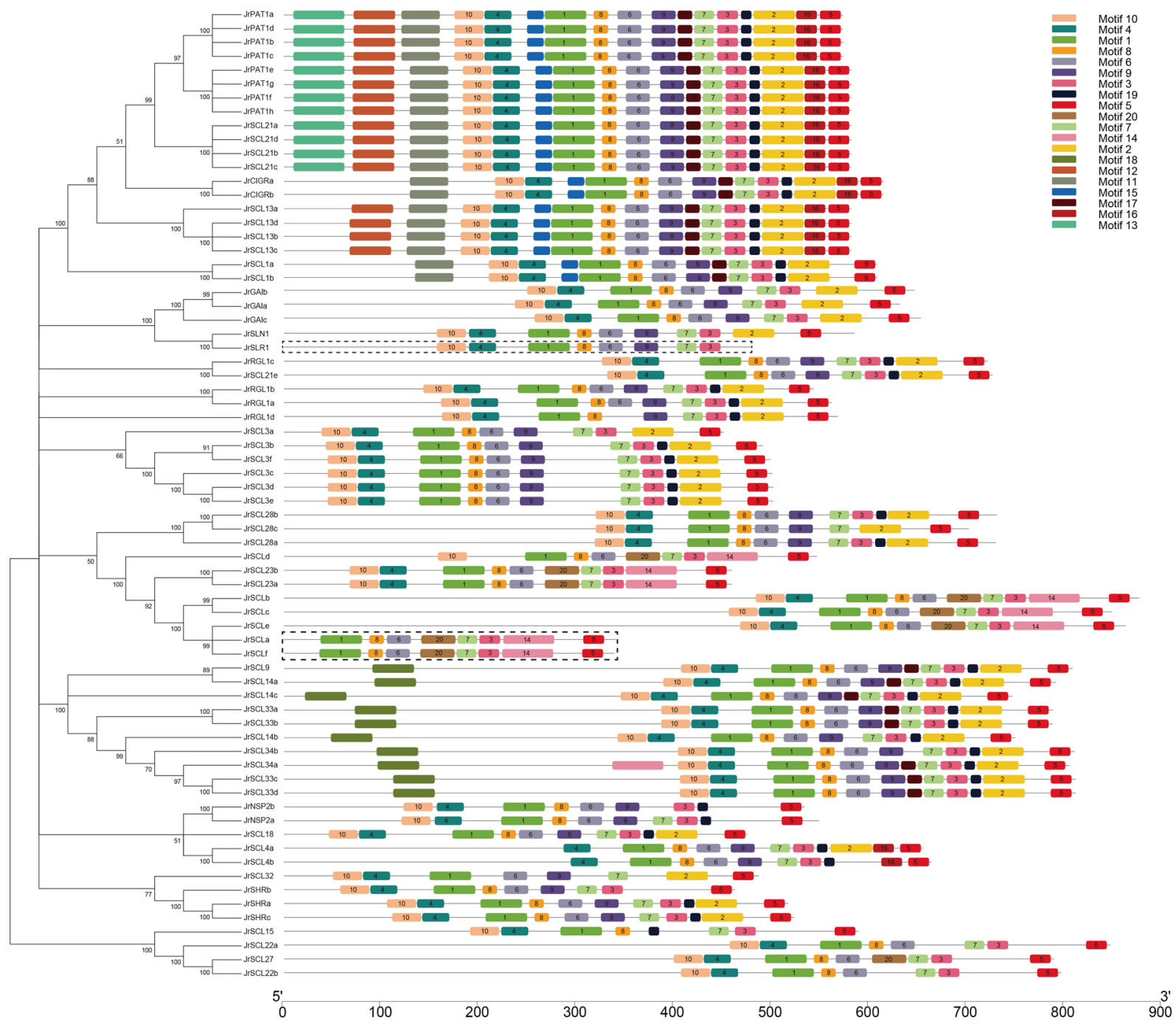

Figure 3. Phylogenetic relationship, motifs and gene structures of GRAS members in walnut.

duplicated gene pairs. What's more, not all of the seven orthologous GRAS genes occurred gene duplication event, two of them (IrSCL15/IrSCL9) showed that they have no duplicated gene pairs in this research.

Expression and function analysis of JrGRAS genes. JrCIGRa-b and JrSCL28a were identified to have a lower expression level in flower bud than that in leaf bud, which suggested these $\operatorname{JrGRAS}$ genes may negatively control the flower buds transition. And expression levels of JrCIGR $a-b$ and JrSCL13b- $d$ were detected up-regulated after flower buds transition (F_3) compared to that in (i) before the flower buds transition (F_1) and (ii) during the flower buds transition (F_2), which indicated that these JrGRAS genes may positively participate in the regulation of walnut flower organs development. Besides, three hub JrGRAS genes (JrSCL3f/JrGAIa/JrSHRc) were predicted by co-expression analysis, which suggested that they may involve in the regulation network of walnut flower buds transition, too.

Functional analysis of the JrGRAS proteins seems to accord with the result of expression analysis. The GRAS domains are interacting with other domains identified by forming the heterodimer or homodimer structure. Up to now, two models of the GRAS domain interacting with other domains have been reported: (i) SHR-SCR heterodimeric structure; (ii) the homodimeric structure of the SCL7 GRAS domain ${ }^{4}$. And in this study, the SCR proteins $(J r S C L a / b / c / d / e / f)$ were predicted to interact with the SHR proteins $(J r S H R a / b / c)$ (Fig. 8), which consist 


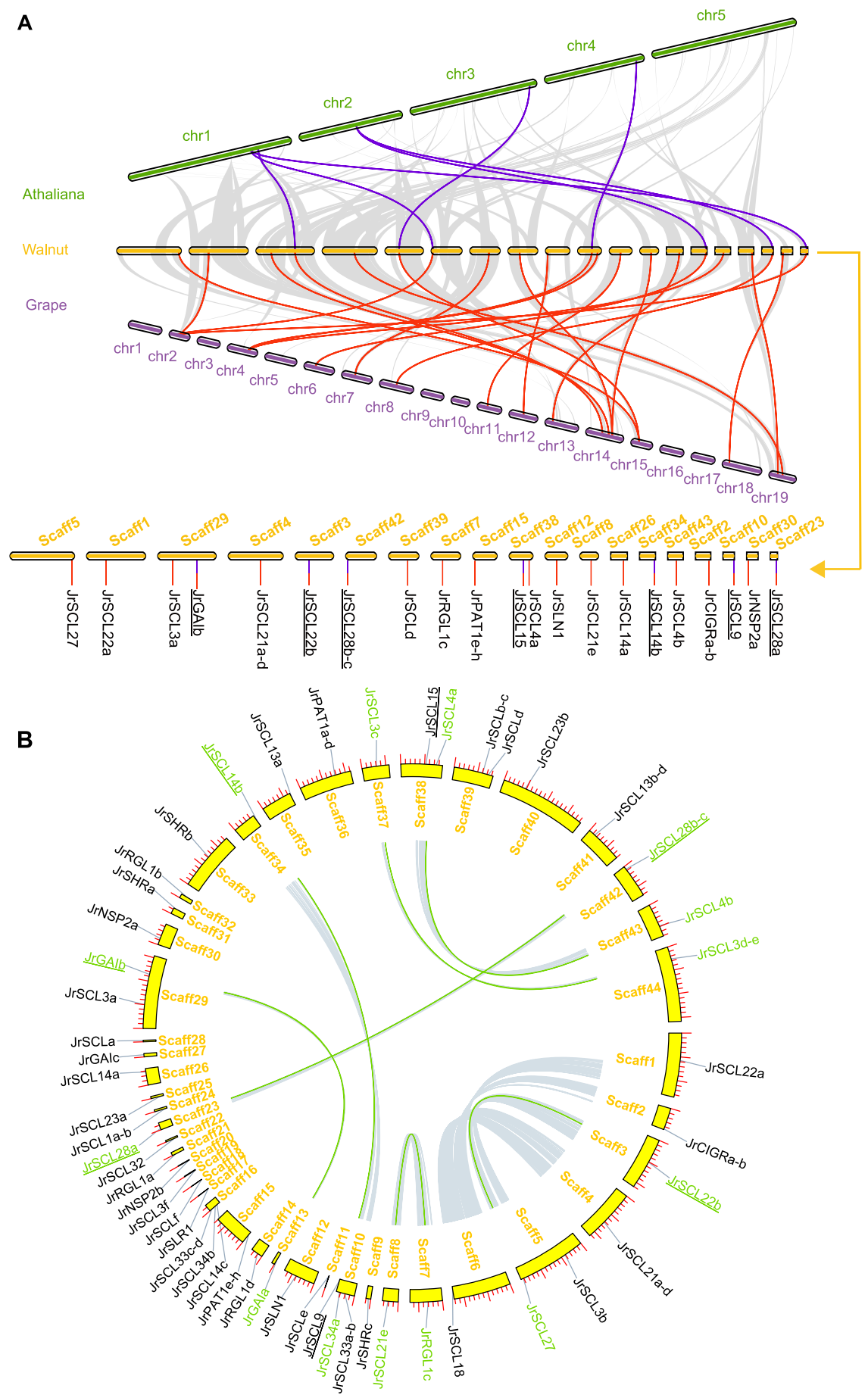

Figure 4. (A) Synteny analysis of GRAS genes between Arabidopsis, walnut and grape. The gray lines in the background indicate the collinear blocks within walnut and other plant genomes, while the blue and red lines highlight the syntenic GRAS gene pairs. (B) Synteny analysis of JrGRAS genes. Gray lines indicate all synteny blocks in the walnut genome, whereas the green lines suggest duplicated GRAS gene pairs. The gene name with an underline means this gene was identified as the synteny gene between different species.

with the SHR-SCR heterodimeric structure model. Besides, protein-protein interaction analysis showed that three hub JrGRASs (JrSCL3f/JrGAIa/JrSHRc) identified by expression analysis also have many interaction partners in the $J r G R A S$ protein-protein interaction network (Fig. 8), these results illustrate how $J r$ GRAS family proteins might form functional complexes, mediating the expression of flower bud transition genes in walnut.

Importantly, the $L A S$ subfamily is involved and necessary in the growth regulation of the meristem formation ${ }^{41,43,44}$. A differentially expressed JrGRAS gene (JrSCL28a) in the LAS subfamily was found expressed both in leaf bud and flower bud, however, its expression level in leaf bud was significantly higher than that in 
A

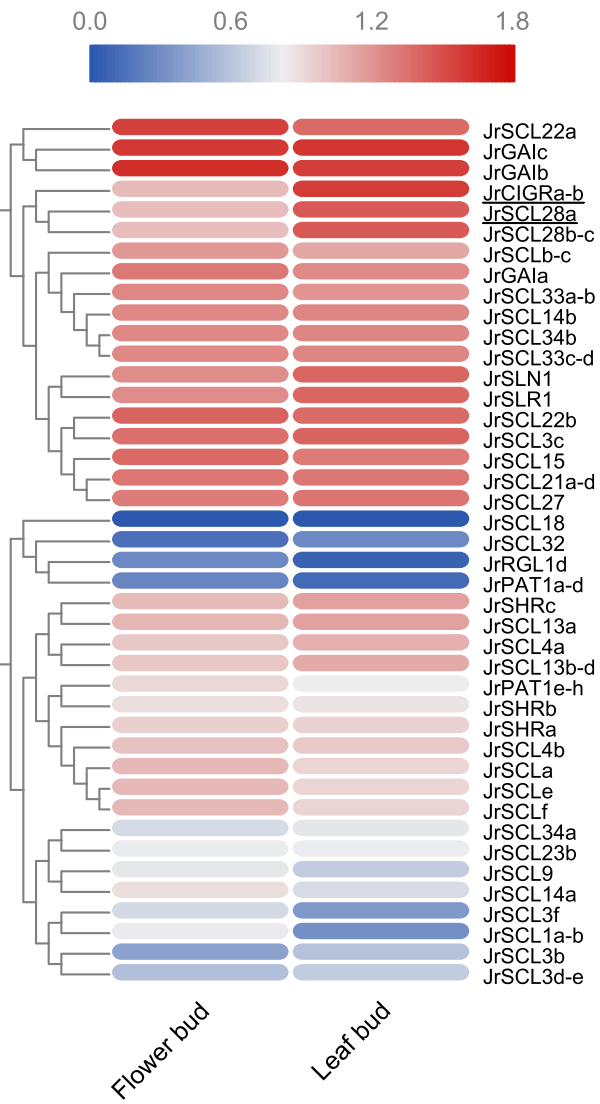

B

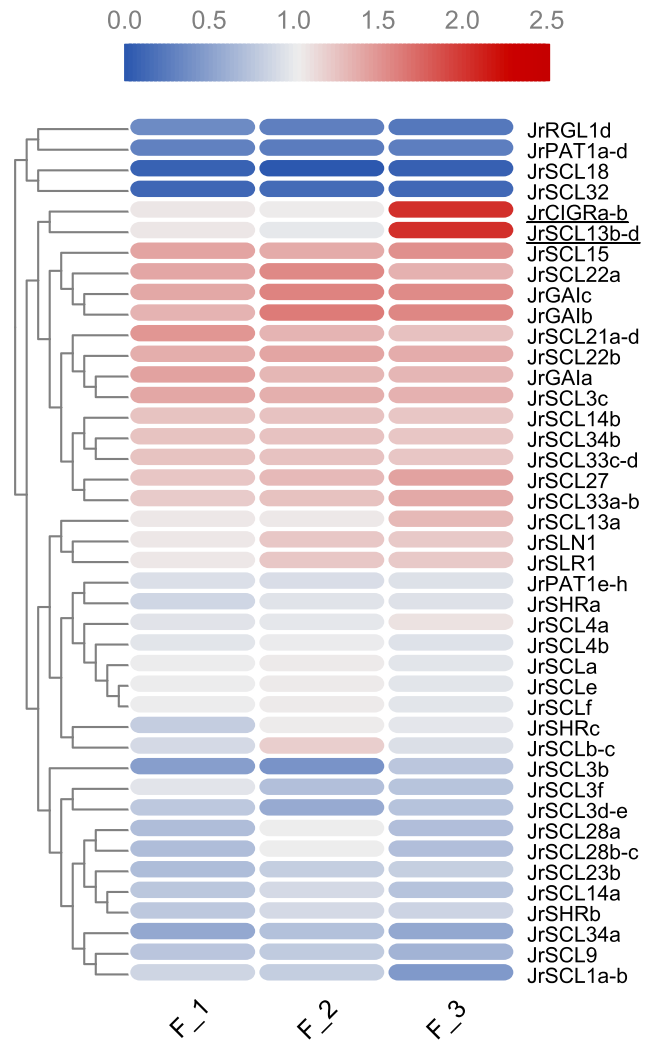

Figure 5. (A) Heatmap of the JrGRAS genes between flower buds and leaf buds. (B) Heatmap of JrGRAS genes expressed differently in three development periods of flower buds (F_1, F_2, and F_3).

flower bud $(\mathrm{P}<0.01)$, the mechanism is still unclear. PAT1 is involved in phytochrome A signal transduction in Arabidopsis $^{35}$. In this study, two DEGs (JrCIGRa- $b$ and $J r S C L 13 b-d, \mathrm{P}<0.01$ ), identified (i) before, (ii) during and (iii) after flower bud transition (F_1, F_2 and F_3), were classified into the PAT subfamily, which indicated light signaling via the phytochrome A photoreceptor controls basic plant developmental processes, including flower bud development. Recently, a single walnut GRAS gene, JrGRAS2 (LOC108996381, JrSCL23b, belongs to the SCL subfamily in this article), was reportedly involved in high-temperature stress tolerance ${ }^{40}$, which offer new insights to the functional diversity of walnut GRAS family members.

In summary, our work laid a foundation for future function investigation of the GRAS members in walnut and provides valuable information about the gene functions of GRAS family in the development of walnut flower bud transition.

\section{Methods}

Identification of GRAS family members in walnut. The latest protein sequences file (GCF_001411555.1_wgs.5d_protein.faa) of walnut genome was downloaded from the NCBI website (ftp://ftp. ncbi.nlm.nih.gov/genomes/all/GCF/001/411/555/GCF_001411555.1_wgs.5d/GCF_001411555.1_wgs.5d_protein.faa.gz). The hmm model of GRAS domain was constructed based on the PF03514 (PFAM website, http:// pfam.xfam.org/family/pf03514) by the hmmbuild program HMMER $3.2^{52}$. Then, we used the hmm model mentioned above to search against the protein databases of walnut genomes with the hmmsearch program in HMMER $3.2^{52}$, the E-value cutoff was $1 \mathrm{e}-10$. The candidate GRAS members were then uploaded to the CD-search website (https://www.ncbi.nlm.nih.gov/Structure/cdd/wrpsb.cgi) to further confirm if they include the proper GRAS domains (sequences included GRAS domain and length of domain sequences was more than $150 \mathrm{aa})$. Gene structures of the JrGRAS genes were drawn by the Biosequence Structure Illustrator program of the TBtools software ${ }^{53}$. Subcellular location information of the JrGRAS proteins was predicted by online software WoLF PSORT II (https://www.genscript.com/wolf-psort.html?src=leftbar).

Multiple alignments and phylogenetic analyses. The domain sequences of the GRAS proteins in Arabidopsis, walnut and grape were downloaded from the Plant Transcription Factor Database (http://planttfdb. cbi.pku.edu.cn/) and aligned using Clustal X $2.1^{54}$. Then these sequences were used to conduct phylogenetic analyses using MEGA 6 software ${ }^{55}$ with 1000 bootstrap replicates. Motifs in the JrGRAS family members were identified by MEME program (http://meme-suite.org/tools/meme) ${ }^{49}$ with a maximum of 20 motifs shown in the result. 


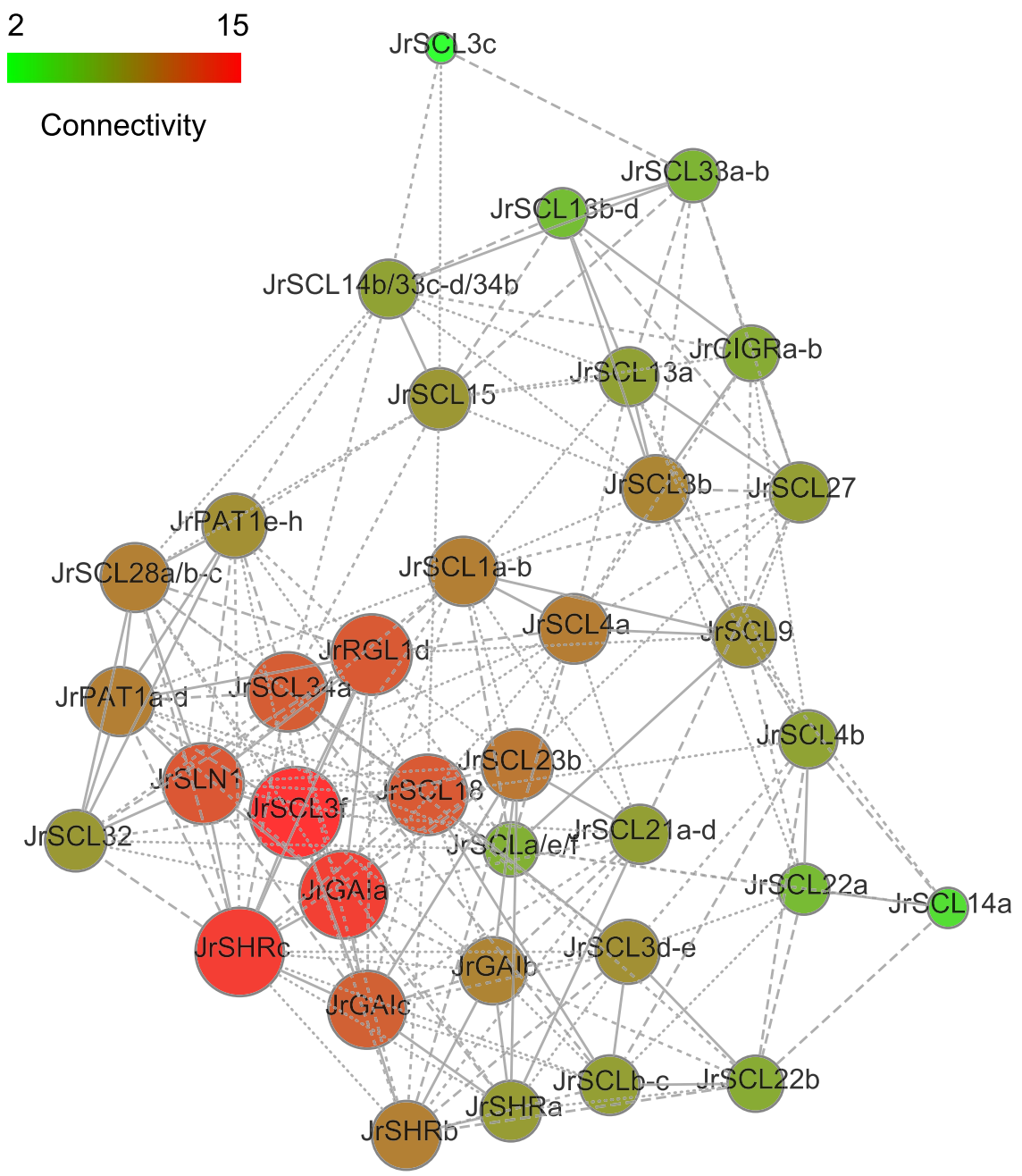

Figure 6. Co-expression networks of $58 \mathrm{JrGRAS}$ genes. In the drawn weight network graph, the weight between genes will be divided into four parts, which are represented by point lines, short dotted lines, long dotted lines and real lines from small to large weights. The larger node and the redder color mean the greater connectivity of the gene in the network graph.

Synteny and gene duplication analysis. Analysis of gene duplication events using MCScanX toolkit ${ }^{56}$, paralogous genes in walnut genome were identified by the duplicate_gene_classifier program with the default parameters of the MCScanX toolkit, and orthologous genes between species were identified by the detect_collinear_tandem_arrays program with the default parameters of the MCScanX toolkit ${ }^{56}$. The genome sequences files and annotation files of Arabidopsis (RefSeq assembly accession: GCF_000001735.2, ftp://ftp.ncbi.nlm. nih.gov/genomes/all/GCF/000/001/735/GCF_000001735.4_TAIR10.1), walnut (RefSeq assembly accession: GCF_001411555.1) and grape (RefSeq assembly accession: GCF_000003745.3, ftp://ftp.ncbi.nlm.nih.gov/ genomes/all/GCF/000/003/745/GCF_000003745.3_12X/) were downloaded from NCBI website (https://www. ncbi.nlm.nih.gov). The circle map of syntenic analysis maps in walnut genome was constructed by TBtools software $^{53}$. Because of the walnut genome was assembled only in scaffold-level, the 44 scaffolds which including the $52 \mathrm{JrGRAS}$ genes were used to represent the walnut genome in the synteny and gene duplication analysis.

Expression analysis of GRAS members. Transcriptome sequencing and library construction were reported in our previous study ${ }^{57}$. Expression analysis of walnut GRAS members was evaluated using the walnut RNA-sequence data among different tissues (leaf bud and female flower bud), development stages (F_1, F_2, F_3). The FPKM values were normalized with the treatment of $\log 10(\mathrm{FPKM})$, and the results were then used to generate heatmap using the HemI software ${ }^{58}$.

RNA isolation and qRT-PCR analysis. The female flower buds were collected before, during, and after flower transition (F_1, F_2 and F_3), and leaf buds were collected during the floral transition period. The Leaf buds and female flower buds $\left(\mathrm{F}_{-} 1, \mathrm{~F}_{-} 2\right.$ and $\left.\mathrm{F}_{-} 3\right)$ were collected and immediately frozen in liquid nitrogen. Total RNA was extracted with RNAout 1.0 (Tianenze, China) as described by the manufacturer and cDNA was reversed reverse-transcribed using the PrimeScript RT Reagent Kit (Takara, China). The real-time PCR analysis 
A
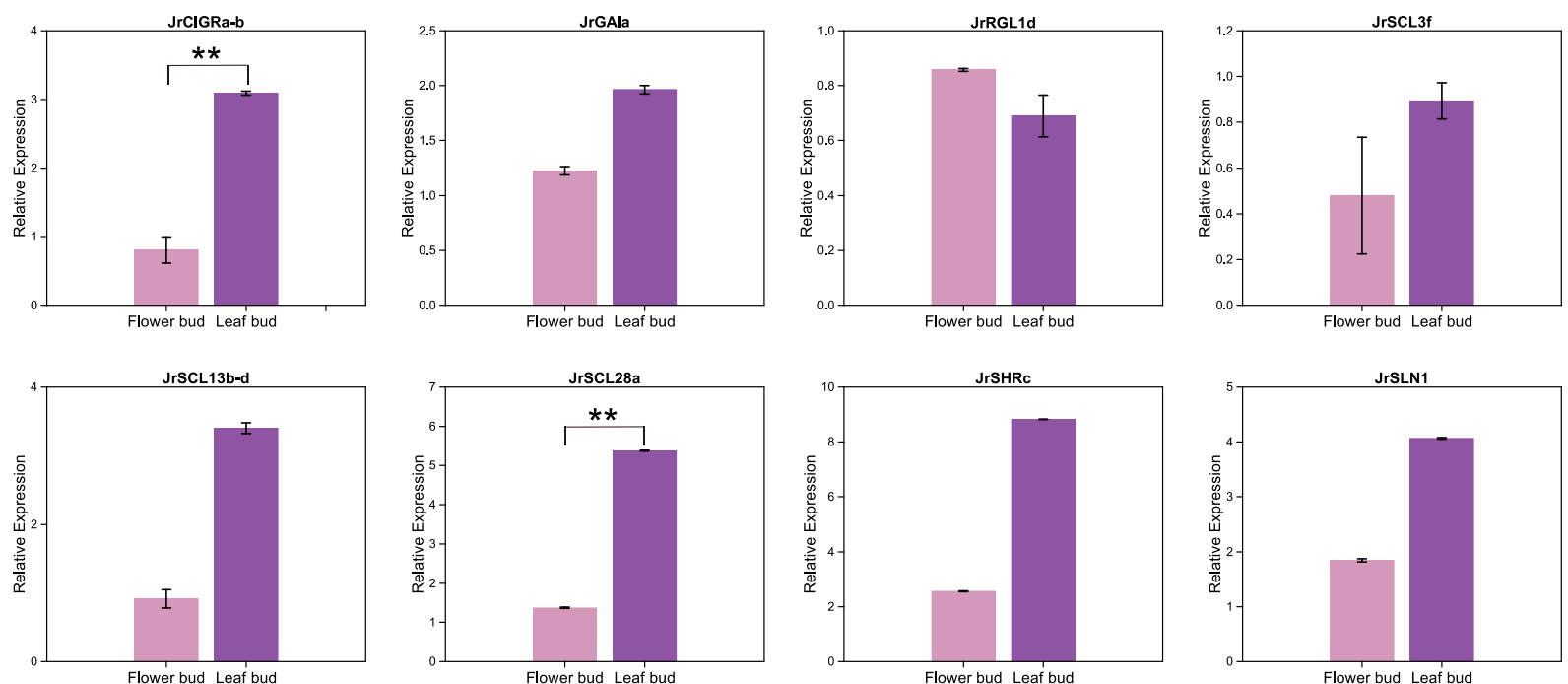

B
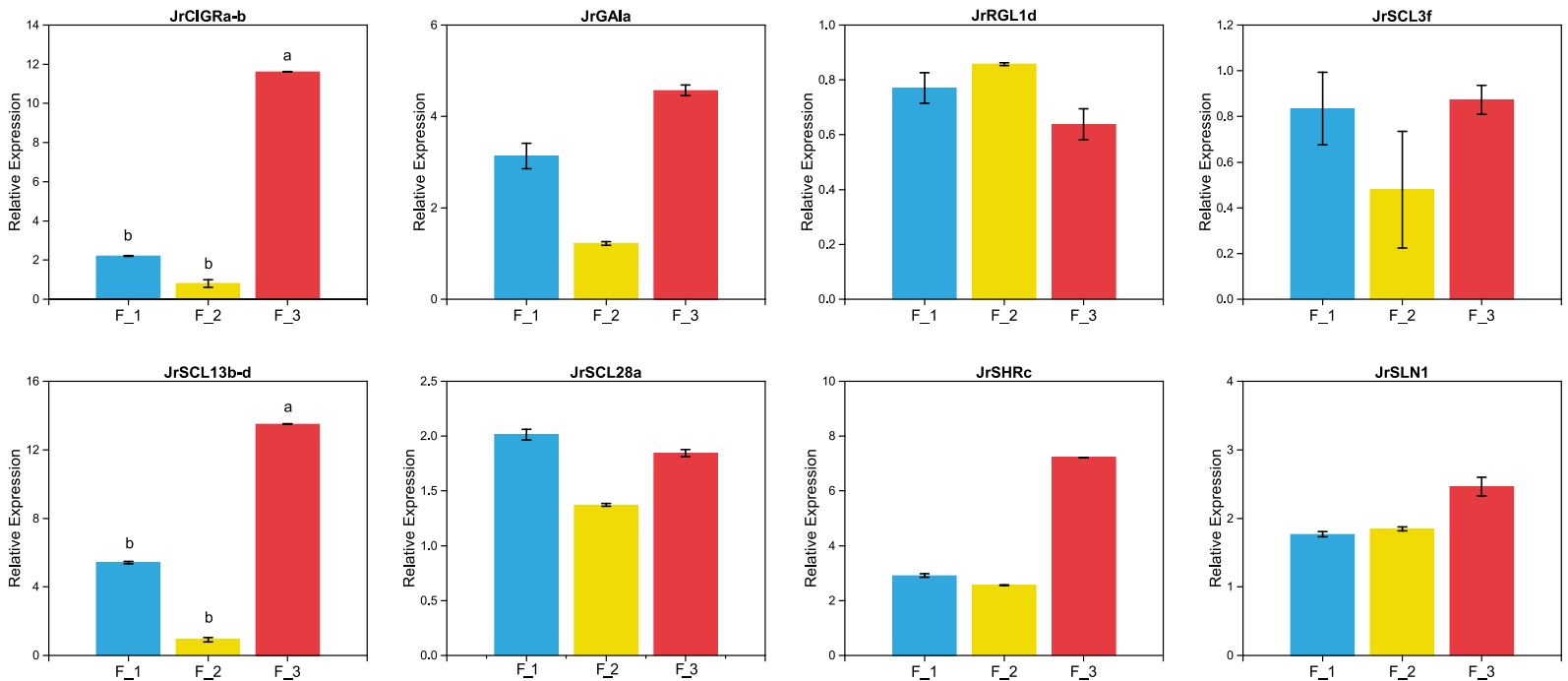

Figure 7. qRT-PCR analysis of JrGRAS genes in different tissues and different development period of flower buds.

was performed using CFX Manager (Bio-Rad, USA) with SYBR Green mixture (Toyobo, Japan), and the walnut actin gene and walnut gadph gene were used for normalization, the amplification was applied using the cycling parameter as described previously ${ }^{45}$. The results were evaluated by the $2^{-\Delta \mathrm{Ct}}$ method according to Livak and Schmittgen ${ }^{59}$.

GO enrichment. The Blast $2 \mathrm{GO}^{60-63}$ software was employed to perform the GO annotation. First, protein sequences of the JrGRAS were used to perform the blastp search against the Swissport database with the E-Value of $1 \mathrm{E}-05$, number of blast hits was 5 . Then the result was conducted a GO mapping, and after that the GO annotation program was used to get the GO annotation of the JrGRAS members. Finally, the GO enrichment analysis was conducted by the online GO enrichment program on the omicshare website (https://www.omicshare.com/ tools/Home/Soft/gogsea).

Interaction network of JrGRAS proteins. The blastp program was used between the walnut GRAS proteins and the Arabidopsis GRAS proteins, each walnut GRAS protein matched a homologous Arabidopsis GRAS protein with the highest score (Table S7). Thirty-three Arabidopsis GRAS proteins which represent the 70 walnut GRAS proteins were uploaded to the String website (https://string-db.org/ $/{ }^{64}$ to predict protein interactions. Except the 33 input proteins, five predicted functional partners of the input proteins were used to construct the network. The walnut GRAS proteins corresponded with the Arabidopsis GRAS proteins are listed below them. The online program ran with default parameters. 


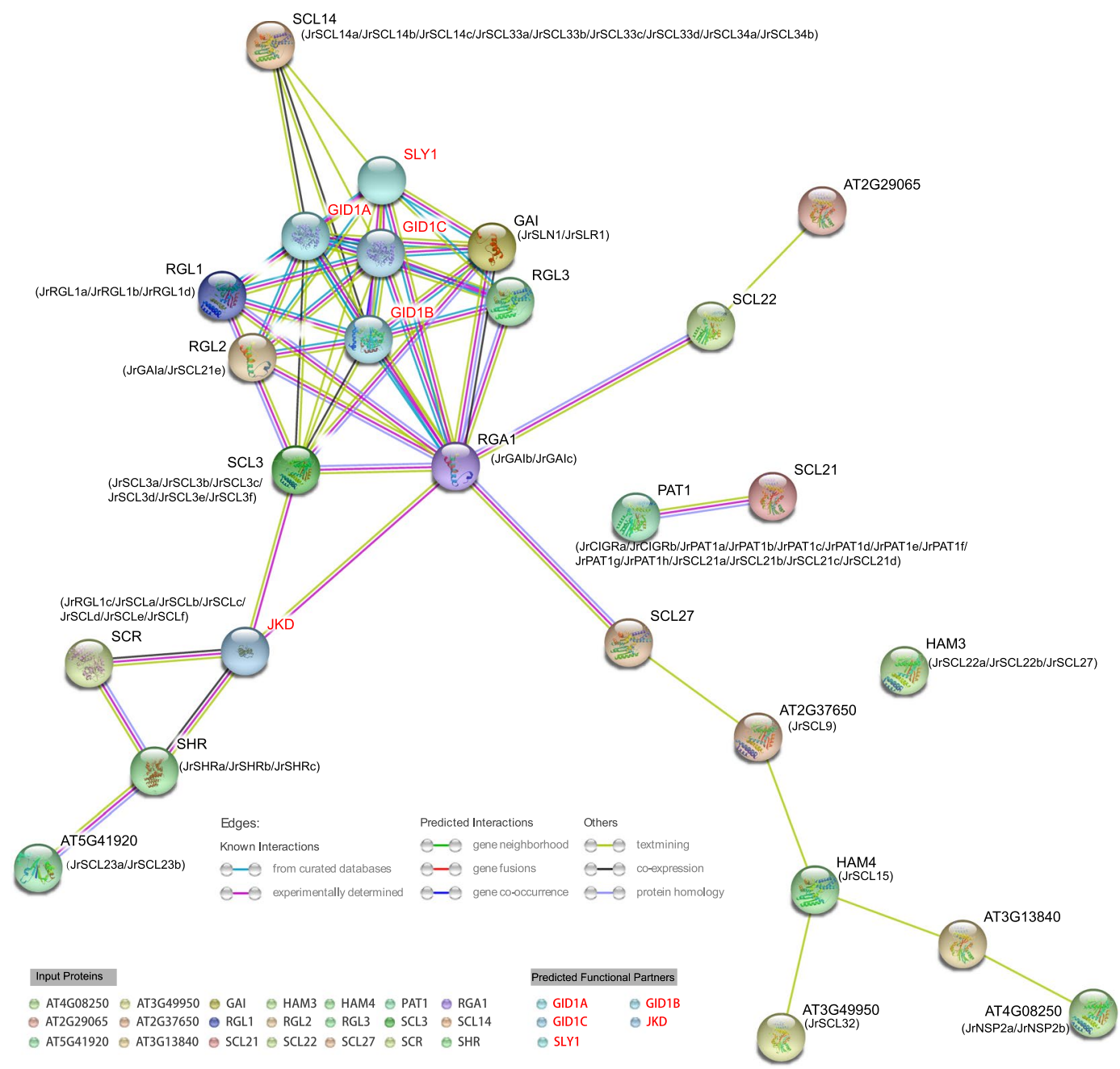

Figure 8. Predicted protein-protein interaction network of $J r G R A S$ proteins. The network nodes represent proteins, the $3 \mathrm{D}$ structure of the proteins is shown inside the nodes and the colors of the line indicate different data sources.

\section{Data Availability}

The data used to support the findings of this study are included in the article.

\section{References}

1. Pysh, L. D., Wysocka-Diller, J. W., Camilleri, C., Bouchez, D. \& Benfey, P. N. The GRAS gene family in Arabidopsis: sequence characterization and basic expression analysis of the SCARECROW-LIKE genes. Plant J 18, 111-119 (1999).

2. Silverstone, A. L., Ciampaglio, C. N. \& Sun, T. The Arabidopsis RGA gene encodes a transcriptional regulator repressing the gibberellin signal transduction pathway. The Plant cell 10, 155-169 (1998).

3. Sun, T. P. The molecular mechanism and evolution of the GA-GID1-DELLA signaling module in plants. Curr Biol 21, R338-345, https://doi.org/10.1016/j.cub.2011.02.036 (2011).

4. Hakoshima, T. Structural basis of the specific interactions of GRAS family proteins. FEBS letters 592, 489-501, https://doi. org/10.1002/1873-3468.12987 (2018).

5. Pauluzzi, G. et al. Surfing along the root ground tissue gene network. Dev Biol 365, 14-22, https://doi.org/10.1016/j. ydbio.2012.02.007 (2012).

6. Petricka, J. J., Winter, C. M. \& Benfey, P. N. Control of Arabidopsis root development. Annu Rev Plant Biol 63, 563-590, https://doi. org/10.1146/annurev-arplant-042811-105501 (2012).

7. Tian, C., Wan, P., Sun, S., Li, J. \& Chen, M. Genome-wide analysis of the GRAS gene family in rice and Arabidopsis. Plant molecular biology 54, 519-532, https://doi.org/10.1023/b:Plan.0000038256.89809.57 (2004).

8. Bolle, C. The role of GRAS proteins in plant signal transduction and development. Planta 218, 683-692, https://doi.org/10.1007/ s00425-004-1203-z (2004).

9. Zhou, S. et al. Manipulation of plant architecture and flowering time by down-regulation of the GRAS transcription factor SIGRAS26 in Solanum lycopersicum. Plant science: an international journal of experimental plant biology 271, 81-93, https://doi.org/10.1016/j. plantsci.2018.03.017 (2018).

10. Sun, X. et al. A functionally required unfoldome from the plant kingdom: intrinsically disordered N-terminal domains of GRAS proteins are involved in molecular recognition during plant development. Plant Mol Biol 77, 205-223, https://doi.org/10.1007/ s11103-011-9803-z (2011).

11. Richards, D. E., Peng, J. \& Harberd, N. P. Plant GRAS and metazoan STATs: one family? Bioessays 22, 573-577, https://doi. org/10.1002/(sici)1521-1878(200006)22:6<573::Aid-bies10>3.0.Co;2-h (2000). 
12. Lee, M. H. et al. Large-scale analysis of the GRAS gene family in Arabidopsis thaliana. Plant molecular biology 67, 659-670, https:// doi.org/10.1007/s11103-008-9345-1 (2008).

13. Day, R. B., Shibuya, N. \& Minami, E. Identification and characterization of two new members of the GRAS gene family in rice responsive to N-acetylchitooligosaccharide elicitor. Biochim Biophys Acta 1625, 261-268 (2003).

14. Liu, X. \& Widmer, A. Genome-wide Comparative Analysis of the GRAS Gene Family in Populus, Arabidopsis and Rice. Plant Molecular Biology Reporter 32, 1129-1145 (2014).

15. Abarca, D. et al. The GRAS gene family in pine: transcript expression patterns associated with the maturation-related decline of competence to form adventitious roots. BMC Plant Biol 14, 354, https://doi.org/10.1186/s12870-014-0354-8 (2014).

16. Song, X. M. et al. Genome-wide analysis of the GRAS gene family in Chinese cabbage (Brassica rapa ssp. pekinensis). Genomics 103, 135-146, https://doi.org/10.1016/j.ygeno.2013.12.004 (2014).

17. Chen, Y. Q. et al. Homology-based analysis of the GRAS gene family in tobacco. Genet Mol Res 14, 15188-15200, https://doi. org/10.4238/2015.November.25.7 (2015).

18. Huang, W., Xian, Z., Kang, X., Tang, N. \& Li, Z. Genome-wide identification, phylogeny and expression analysis of GRAS gene family in tomato. BMC Plant Biol 15, 209, https://doi.org/10.1186/s12870-015-0590-6 (2015).

19. Niu, Y., Zhao, T., Xu, X. \& Li, J. Genome-wide identification and characterization of GRAS transcription factors in tomato (Solanum lycopersicum). PeerJ 5, e3955, https://doi.org/10.7717/peerj.3955 (2017).

20. Lu, J., Wang, T., Xu, Z., Sun, L. \& Zhang, Q. Genome-wide analysis of the GRAS gene family in Prunus mume. Mol Genet Genomics 290, 303-317, https://doi.org/10.1007/s00438-014-0918-1 (2015).

21. Wu, Z. Y. et al. Genome-wide analysis of the GRAS gene family in physic nut (Jatropha curcas L.). Genet Mol Res 14, 19211-19224, https://doi.org/10.4238/2015.December.29.31 (2015).

22. Xue, L. et al. Network of GRAS transcription factors involved in the control of arbuscule development in Lotus japonicus. Plant Physiol 167, 854-871, https://doi.org/10.1104/pp.114.255430 (2015).

23. Grimplet, J., Agudelo-Romero, P., Teixeira, R. T., Martinez-Zapater, J. M. \& Fortes, A. M. Structural and Functional Analysis of the GRAS Gene Family in Grapevine Indicates a Role of GRAS Proteins in the Control of Development and Stress Responses. Front Plant Sci 7, 353, https://doi.org/10.3389/fpls.2016.00353 (2016).

24. Sun, X. et al. A characterization of grapevine of GRAS domain transcription factor gene family. Funct Integr Genomics 16, 347-363, https://doi.org/10.1007/s10142-016-0479-y (2016).

25. Zhao, H. et al. Comprehensive analysis of multi-tissue transcriptome data and the genome-wide investigation of GRAS family in Phyllostachys edulis. Sci Rep 6, 27640, https://doi.org/10.1038/srep27640 (2016).

26. Xu, W. et al. Genome-Wide Identification, Evolutionary Analysis, and Stress Responses of the GRAS Gene Family in Castor Beans. Int J Mol Sci 17, https://doi.org/10.3390/ijms17071004 (2016).

27. Yang, C. J., Li, G. Y. \& Cui, Y. L. Bioinformatic analyses of GRAS genes in Betula kirghisorum based on transcriptome data. Genet Mol Res 15, https://doi.org/10.4238/gmr.15038321 (2016).

28. Zhang, L., Li, Q., Chen, J. F. \& Chen, W. S. Computational identification and systematic classification of novel GRAS genes in Isatis indigotica. Chinese journal of natural medicines 14, 161-176, https://doi.org/10.1016/s1875-5364(16)30013-9 (2016).

29. Fan, S. et al. Identification, Classification, and Expression Analysis of GRAS Gene Family in Malus domestica. Frontiers in physiology 8, 253, https://doi.org/10.3389/fphys.2017.00253 (2017).

30. Guo, Y. et al. Identification and expression of GRAS family genes in maize (Zea mays L.). Plos One 12, e0185418, https://doi. org/10.1371/journal.pone.0185418 (2017).

31. Zhang, H. et al. Genome-wide characterization of GRAS family genes in Medicago truncatula reveals their evolutionary dynamics and functional diversification. Plos One 12, e0185439, https://doi.org/10.1371/journal.pone.0185439 (2017).

32. Wang, Y. X. et al. Genome-wide identification and expression analysis of GRAS family transcription factors in tea plant (Camellia sinensis). Sci Rep 8, 3949, https://doi.org/10.1038/s41598-018-22275-z (2018).

33. Zhang, B. et al. Genome-wide analysis of GRAS transcription factor gene family in Gossypium hirsutum L. BMC Genomics 19, 348, https://doi.org/10.1186/s12864-018-4722-x (2018).

34. Peng, J. et al. The Arabidopsis GAI gene defines a signaling pathway that negatively regulates gibberellin responses. Gene Dev 11, 3194-3205, https://doi.org/10.1101/gad.11.23.3194 (1997).

35. Bolle, C., Koncz, C. \& Chua, N. H. PAT1, a new member of the GRAS family, is involved in phytochrome A signal transduction. Genes Dev 14, 1269-1278 (2000).

36. Torres-Galea, P., Hirtreiter, B. \& Bolle, C. Two GRAS proteins, Scarecrow-Like21 and Phytochrome a Signal Transduction1, function cooperatively in phytochrome A signal transduction. Plant Physiol 161, 291-304, https://doi.org/10.1104/pp.112.206607 (2013).

37. Mayrose, M., Ekengren, S. K., Melech-Bonfil, S., Martin, G. B. \& Sessa, G. A novel link between tomato GRAS genes, plant disease resistance and mechanical stress response. Molecular plant pathology 7, 593-604, https://doi.org/10.1111/j.1364-3703.2006.00364.x (2006).

38. Yuan, Y. et al. Overexpression of VaPAT1, a GRAS transcription factor from Vitis amurensis, confers abiotic stress tolerance in Arabidopsis. Plant Cell Rep 35, 655-666, https://doi.org/10.1007/s00299-015-1910-x (2016).

39. $\mathrm{Xu}, \mathrm{K}$. et al. OsGRAS23, a rice GRAS transcription factor gene, is involved in drought stress response through regulating expression of stress-responsive genes. BMC Plant Biol 15, 141, https://doi.org/10.1186/s12870-015-0532-3 (2015).

40. Yang, G. et al. The walnut transcription factor JrGRAS2 contributes to high temperature stress tolerance involving in Dof transcriptional regulation and HSP protein expression. BMC Plant Biol 18, 367, https://doi.org/10.1186/s12870-018-1568-y (2018).

41. Stuurman, J., Jaggi, F. \& Kuhlemeier, C. Shoot meristem maintenance is controlled by a GRAS-gene mediated signal from differentiating cells. Genes Dev 16, 2213-2218, https://doi.org/10.1101/gad.230702 (2002).

42. Li, X. et al. Control of tillering in rice. Nature 422, 618-621, https://doi.org/10.1038/nature01518 (2003).

43. Greb, T. et al. Molecular analysis of the Lateral Suppressor gene in Arabidopsis reveals a conserved control mechanism for axillary meristem formation. Genes Dev 17, 1175-1187, https://doi.org/10.1101/gad.260703 (2003).

44. Engstrom, E. M. et al. Arabidopsis homologs of the petunia hairy meristem gene are required for maintenance of shoot and root indeterminacy. Plant Physiol 155, 735-750, https://doi.org/10.1104/pp.110.168757 (2011).

45. Quan, S. et al. Identification and characterization of NF-Y gene family in walnut (Juglans regia L.). BMC Plant Biol 18, 255, https:// doi.org/10.1186/s12870-018-1459-2 (2018).

46. Pollegioni, P. et al. Rethinking the history of common walnut (Juglans regia L.) in Europe: Its origins and human interactions. Plos One 12, e0172541, https://doi.org/10.1371/journal.pone.0172541 (2017).

47. Abdallah, I. B. et al. Content of carotenoids, tocopherols, sterols, triterpenic and aliphatic alcohols, and volatile compounds in six walnuts (Juglans regia L.) varieties. Food Chemistry 173, 972-978 (2015).

48. Martinez-Garcia, P. J. et al. The walnut (Juglans regia) genome sequence reveals diversity in genes coding for the biosynthesis of nonstructural polyphenols. Plant J 87, 507-532, https://doi.org/10.1111/tpj.13207 (2016).

49. Bailey, T. L. et al. MEME SUITE: tools for motif discovery and searching. Nucleic acids research 37, W202-208, https://doi. org/10.1093/nar/gkp335 (2009).

50. Presson, A. P. et al. Integrated Weighted Gene Co-expression Network Analysis with an Application to Chronic Fatigue Syndrome. BMC Systems Biology 2, 95-95, https://doi.org/10.1186/1752-0509-2-95 (2008).

51. Prince, V. E. \& Pickett, F. B. Splitting pairs: the diverging fates of duplicated genes. Nat Rev Genet 3, 827-837, https://doi.org/10.1038/ $\operatorname{nrg} 928(2002)$. 
52. Eddy, S. R. Profile hidden Markov models. Bioinformatics 14, 755-763 (1998).

53. Chen, C., Xia, R., Chen, H. \& He, Y. TBtools, a Toolkit for Biologists integrating various HTS-data handling tools with a userfriendly interface, https://doi.org/10.1101/289660 (2018).

54. Thompson, J. D., Gibson, T. J. \& Higgins, D. G. Multiple sequence alignment using ClustalW and ClustalX. Current protocols in bioinformatics Chapter 2, Unit 23 , https://doi.org/10.1002/0471250953.bi0203s00 (2002).

55. Tamura, K., Stecher, G., Peterson, D., Filipski, A. \& Kumar, S. MEGA6: Molecular Evolutionary Genetics Analysis version 6.0. Mol Biol Evol 30, 2725-2729, https://doi.org/10.1093/molbev/mst197 (2013).

56. Wang, Y. et al. MCScanX: a toolkit for detection and evolutionary analysis of gene synteny and collinearity. Nucleic acids research 40, e49, https://doi.org/10.1093/nar/gkr1293 (2012).

57. Quan, S. et al. Stages identifying and transcriptome profiling of the floral transition in Juglans regia. Sci Rep 9, 7092, https://doi. org/10.1038/s41598-019-43582-z (2019).

58. Deng, W., Wang, Y., Liu, Z., Cheng, H. \& Xue, Y. HemI: a toolkit for illustrating heatmaps. Plos One 9, e111988, https://doi. org/10.1371/journal.pone.0111988 (2014).

59. Livak, K. J. \& Schmittgen, T. D. Analysis of relative gene expression data using real-time quantitative PCR and the 2(-Delta Delta C(T)) Method. Methods (San Diego, Calif.) 25, 402-408, https://doi.org/10.1006/meth.2001.1262 (2001).

60. Gotz, S. et al. High-throughput functional annotation and data mining with the Blast2GO suite. Nucleic acids research 36, 3420-3435, https://doi.org/10.1093/nar/gkn176 (2008).

61. Gotz, S. et al. B2G-FAR, a species-centered GO annotation repository. Bioinformatics 27, 919-924, https://doi.org/10.1093/ bioinformatics/btr059 (2011).

62. Ana, C. \& Stefan, Gt Blast2GO: A Comprehensive Suite for Functional Analysis in Plant Genomics. International Journal of Plant Genomics 2008, 619832 (2008).

63. Conesa, A. et al. Blast2GO: a universal tool for annotation, visualization and analysis in functional genomics research. Bioinformatics 21, 3674-3676, https://doi.org/10.1093/bioinformatics/bti610 (2005).

64. Szklarczyk, D. et al. The STRING database in 2017: quality-controlled protein-protein association networks, made broadly accessible. Nucleic acids research 45, D362-D368, https://doi.org/10.1093/nar/gkw937 (2017).

\section{Acknowledgements}

This work was supported by the important National Science and Technology Specific projects of Xinjiang (No. 201130102-1-4) and the National Natural Science Foundation of China (No. 30560090).

\section{Author Contributions}

J.X.N. led and coordinated the project, J.X.N. and S.W.Q. designed the study, S.W.Q., L.Z., H.X., L.M. and Y.Q. collected the plant materials and isolated the RNA. S.W.Q. and L.Z. conducted the real-time quantitative PCR. S.W.Q. conducted the bioinformatics analysis and wrote the paper. All authors have read and agree with the final manuscript. J.X.N. is the corresponding author and is responsible for all contact and correspondence.

\section{Additional Information}

Supplementary information accompanies this paper at https://doi.org/10.1038/s41598-019-48287-x.

Competing Interests: The authors declare no competing interests.

Publisher's note: Springer Nature remains neutral with regard to jurisdictional claims in published maps and institutional affiliations.

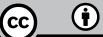

Open Access This article is licensed under a Creative Commons Attribution 4.0 International License, which permits use, sharing, adaptation, distribution and reproduction in any medium or format, as long as you give appropriate credit to the original author(s) and the source, provide a link to the Creative Commons license, and indicate if changes were made. The images or other third party material in this article are included in the article's Creative Commons license, unless indicated otherwise in a credit line to the material. If material is not included in the article's Creative Commons license and your intended use is not permitted by statutory regulation or exceeds the permitted use, you will need to obtain permission directly from the copyright holder. To view a copy of this license, visit http://creativecommons.org/licenses/by/4.0/.

(C) The Author(s) 2019 